\title{
Calidad y Fiabilidad en la Proyección de Plantas Industriales
}

\author{
Gilberto D. Hernández Pérez
}

Dr. Ing. Universidad Técnica "Otto von Guerickc", Magdeburg, R.F.A.

Profesor Titular y Jefe del Dpto. de Ing. Industrial de la Universidad Central de Las Villas.

\section{Ramón A. Pons Murguía}

Ing. Industrial, Profesor Auxiliar y Jefe del Grupo de Aseguramiento de la Calidad del Dpto. de Ing. Industrial de la Universidad Central de Las Villas.

\section{Roberto Cespón Castro}

Ing. Industrial, Profesor Asistente del Dpto. de Ing. Industrial de la Universidad Central de Las Villas.

Carretera a Camajuani Km 5 1/2. Santa Clara/Villa Clara, República de Cuba.

Código Postal: 54830. Telf. 81272. Fax: (052-422-8-16-()8.

Palabras Claves: Proyección de instalaciones fabriles, aseguramiento de la calidad y calidad en la proyección de plantas industriales.

Key words: Industrial plants design, quality assurance and reliability of industrial plants design.

\section{RESUMEN}

El proyecto de una planta industrial y de sus instalaciones constituye una previsión de la producción que en éstas se llevará a cabo; es tambien un producto al que se asocian requisitos de calidad y liabilidad al igual que a los productos finales que saldrán de sus instalaciones. Sin embargo. no siempre este enfoyue se corresponde con el control dela calidad de los proyectos en las metodologias y regulaciones vigentes. El presente trabajo representa una contribución metodológica al aseguramiento de la calidad de este complejo producto que es una planta industrial. con un enfoque moderno para yue sirva de base al perfeccionamiento de este complejo processso.

\section{ABSTRACT}

The following work is aimed to describe a modern approach for quality assurance of industrial plant. design by. means of a methodological contribution that can be taken as a basis for design improvement. Key questions whose answers are the basis for a proper quality assurance approach oriented to this complex process are described. Alain quality specifications are pointed out. Finally. de sign process errors and theirclassified causes, quality improvement oriented countermeasures as well as reliability considerations and quality evaluation of design are also described. 
- La calidad de quć elementos o sistemnas parciales determinan en lo fundamental la calidad del provecto en su conjunto, visto incluso en su interralación con el entorno económico-social?

\section{Introducción}

- Cómo y dónde pueden aparecer los crrores durante el proceso de proyección y como pueden evitarse, climinarcs o reducirse éstos?

Al proceso industrial para la producción de bienes materiales le es inherente un alto grado de incstabilidad, dado por una progresiva precisión de la interrelación entre los procesos de claboración, trransformación, ensamblaje, transporte, almaccnaje y control en todas las fases del proceso de reproducción empresarial (preparación, cjecución y venta de la producción).

El cfecto útil de la mayoria de las instalaciones industriales crece con la calidad que particularmente se logra en las actividades de preparación de la producción, especialmentc en aquellas vinculadas a la proyección de las nuevas capacidades y la "reconstrucción" (1) de las ya cxistentes.

La calidad de la produccion debe propiciar cl mayor efecto útil posible, no solo de la instalación fabril en particular sino tambien a nivel territorial y de la economia nacional. Esto último resulta de vital importância en las concepciones de desarrollo de nuestras economias ante importancia cn las concepciones de desarrollo de nuestras cconomías ante el reto de un mundo cada vez más competitivo.

Un adccuado enfoque del aseguramiento de la calidad de los proyectos de plantas industriales debe plantearse y dar respuesta a las interrogantes fundamentales siquientes:
- Qué medidas de control deben acompanar al proceso de proyeccion y cómo deben éjecutarse racionalmente éstas?

- Qué medidas deben ser tomadas en el proyecto para garantizar una instalación industrial confiable?

A pesar de que regularmente en toda actividad de provecto se garantiza un determinado nível de respuesta a cstas interrogantes a través de las metodologias y regulaciones vigentes y en utilización en nucstros paises. no siempre los resultados obtenidos se corresponden con las expectativas. fundamentalmente desde cl punto de vista cualitativo. Sin dudas, a cstas deficiencias se asocian problemas de enfoque, integralidad profundidad y racionalidad en la concepción y aplicación de estas metodologias y regulaciones a casos concretos.

El presente trabajo representa una contribución metodológica al aseguramiento de la calidad de este "complejo producto" que es una planta industrial, con un enfoque moderno para que sirva de base al perceccionamiento de la actividad inversionista en nuestros paises y con ello a la aplicación más efectiva del concepto de "Calidad Total" en csta importante esfera de trabajo. 


\section{Calidad de un Proyecto,} Requerimientos,

\section{Características y}

\section{Especificaciones de}

\section{Calidad de los}

\section{Proyectos}

Definiciones tales como "Conformidad para el uso", "conformidad con las especificaciones" "satisfacción de expectativas del consumidor", "asociación con las pérdidas que un producto imprime a la sociedad después de su venta", "excelencia" y "lo que el consumidor desea adquirir", han sido utilizadas por diferentes autores o asociaciones y adoptadas como tal para diferentes propósitos por publicistas, ingenicros y otros profesionales vinculados al desarrollo de productos para definir el concepto de calidad de un producto o servicio y a partir de éstas los de requerimientos, características y especificaciones de calidad. Aún cuando cac a definición de calidad puede presentar particularidades e interpretaciones que las adecuan en mayor o menor grado a uno u otro objetivo o fín (ver, por ejemplo 1), Crosby 3 plantes la no existencia de grandes diferencias de contenido en las mismas y si destaca la necesidad de determinar con precisión los requerimientos, características y especificaciones de calidad que las asocian a un producto o servicio en especifico. Lo anteriormente expuesto es válido también para el caso del proyecto de una instalación industrial - considerada con un "producto complejo" como paso previo y necesario para la definición del concepto de calidad de un proyecto "Critério sobre la totalidad de propiedades de un proyecto y el grado de adecuación o concordancia con el objetivo, fin o función prevista para éste (Rockstroh, 10 , así como del de sus requerimientos y caracteristicas de calidad, y de sus correspondientes cspccificaciones sin enbargo, para poder alcanzar las altos niveles de calidad exigidos en la proyección de instalaciones fabriles en nuestros dias ticnen que ser satsifechas diferentes funciones parciales que se constituyen a su vez, en premisas de la actividad o proceso de proveccion; entre las principales se encuentran:

- la consideración de la ley de economia del tiempo en la proyección. cjucución y puesta en marcha de las instalaciones objeto de inversión (cumplimiento de los cronogramas correspondientes):

- el alcance de una clevada productividad del trabajo y rendimiento de las instalaciones en los marcos de una determinada y necesaria Mcxibilidad tecnológica, capacitiva y estructural (ver 18):

- la obteción de una adecuada economia material y de una clevada eficiencia energética, asi como de una flexibilidad y confiabilidad racional en la proyccción de los sistemas de Manipulación - Almacenaje-Transportc (M.A.T)) y de generación y' distribución de cnergia y portadores cnergéticos:

- el logro de un ambiente de trabajo confortable. higienico y scguro para el hombre que labora en el proceso de producción, así como de una clevada calidad de la protección de las instalaciones y del medio ambiente.

El proyecto de una instalación industrial, como tal. cs la previsión de una producción, es también, y por analogia, un producto complejo, al que se asocian niveles, requerimientos, características, especificaciones e indices de calidad y fiabilidad al iqual que a los produc- 
tos finales que saldrán de las futuras instalaciones que son objeto de proyección.

En analogia de lo planteado por Almeida $Y$ Tolcdo 1. el nivel de calidad total de una variante de solución de proyecto " $k$ " $\left(Q_{k}\right)$, puede ser interpretada a través de la función. (1)

$$
Q_{k}=f\left(Q_{i}\right)=\sum_{i=1}^{i} k_{i}{ }^{*} Q_{i}
$$

donde:

$\mathrm{Q}_{\mathrm{i}}=$ indices relativos de calidad de una variante de solución de proyecto que resultan de la comparación de sus indices de calidad reales con los índices de calidad de una solución de proyecto progressiva tomada como referência;

$\mathrm{K}_{\mathrm{i}}=$ indices de ponderación que expresan la influcncia que ejerce cada índices de calidad en la calidad total de la solución de proyecto:

$\mathrm{i}=$ variable de conteo de los indices de calidad considerados como fundamentales en la solución de proyccto; $\mathrm{i}=1$ (1) $n$.

Mientras que los índices relativos de calidad $\left(Q_{i}\right)$ constituycn la expresión cuantitativa de la caracteristica de calidad " $i$ " asociada con la calidad de la variante de solución de proyecto " $\mathrm{k}$ ", los indices de ponderación $\left(\mathrm{K}_{\mathrm{i}}\right)$ son proporciones estimadas por diferentes métodos (por ejemplo, métodos de expertos) para quantificar el grado de influencia de cada característica en la calidadd total de la variante de solución de proyecto que se trate. De esta forma, tiene que cumplirse que:

$$
\sum_{i=1}^{i} k_{i}=1.0
$$

Segun Juran |7|, "cualquicr rasgo distintivo (propicdad, atributo, etc.) de un producto, material o proceso que necesite para lograr la concordancia de éste con el uso a que se destina constituye a una característica de calidad". Las caracteristicas de calidad de los proycctos se derivan, a su vez, de los requerimientos o exigencias que se inponen a éstos. Entre los principales requerimientos de calidad y de consideración más universal en los proyectos de plantas industriales, derivadas a su vez, de las premisas del proceso de proyección antes senaladas, se encuentran:

- clevado rendimiento productivo; o sea, una proporción adecuada entre los necesarios gastos de trabajo vivo y pretérito y los resultados del proceso de producción durante el período de su vida útil;

- máxima funcionabilidad: o sea, el aseguramicnto de la continuidad temporal del proceso de trabajo proyectado para las máquinas, equipos e instalaciones de todo tipo considerado en el proyecto como función de la disponibilidad, frabilidad, mantenibilidad y durabilidad individual de las mismas e integralmente del sistema en su conjunto;

- alta flexibilidad y capacidad de reacción: o sea, capacidad de adaptación y respuesta del sistema ante las influencias de carácter dinámico y estocástico provocados por variaciones o cambios con los programas de producción de forma racional, económica, agil y rápida;

- decuada fiabilidad del sistema en su conjunto, como función de la fiabilidad indi- 
vidual de los elementos y sistemas parciales que lo componen durante su vida útil;

- elevado grado de utilización del volumen constructivo y del área disponible;

- consumo racional de materiales (principales y auxiliares), herramental, energia y portadores energéticos;

- conformación racional de los flujos materialaes y de personal, así como energéticos e informacionales en el território de la fábrica, asi como de los sistemas e instalaciones correspondientes;

- conformacións racional del ambiente de trabajo en los puestos y áreas de trabajo, sectores productivos, talleres y su general de la fabrica, asi como una elevada calidad en la estética, "confort", seguridad y protección de las instalaciones y del medio ambiente;

- impacto económico favorable; comprobado a través de los correspondientes parámetros e índices técnico-económicos fijados para sua evaluación.

Los requerimientos anteriormente enunciados en forma sintética y que permiten "caracterizar" la calidad de un proyecto de forma general, tienen que ser "convertidas" sucesivamente en características y en especificaciones de calidad para poder lograr una evaluación racional de una magnitud compleja como lo es la calidad del proyecto de una instalación industrial en las diferentes fases y etapas de su elaboración. Esta "conversión" de los requerimientos en características y especificationes de calidad para el proyecto no es una tarea fácil y debe llevarse a cabo a través de una precisión de estos requerimientos $\mathrm{u}$ objetivos a alcanzar en el proyecto y de los niveles de calidad asociados a éste que pueden ser objetivamentés alcanzados, mediante la fijación de forma concreta en el planteamiento de la tarea de parámetros, índices, cualidades, etc., que deben ser satisfechos tanto en la documentación de un proyecto, cono en las fases subseguientes de construcción, montage, pucsta en marcha y funcionamiento de las instalaciones durante sua vida útil. Esto debe ser responsabilidad directa del inversionista en estrecha cordinación con las restantes entidades que participan en la inversión e incluidas como obligaciones de las partes correspondientes en los respectivos contratos.

\section{Errores en el Proceso de Proyección, Tipos y Causas que los Provocan Vías para Evitarlos, Elimnarlos y/o Reducir sus Efectos}

La obtcnción de un nivel de calidad elevado en un proyecto, ya sea el correspondiente a la creación de nuevas capacidades productivas como de la reconstrucción de plantas industriales ya existentes e incluso, para la ejecucion de una determinada medida o solución racionalizadora de cierta envergadura presupone

- evitar los errores que pueden presentarse directamente en la solución de una determinada tarea de proyecto;

- eliminar los errores y/o reducir sus efectos que, no obstante, pueden presentarse 
al no considerar entre otras causas, el proceso de producción en su dinámica a partir de su puesta en marcha.

Estos errores se presentam en el proceso de proyección debido fundamentalmente a tres causas:

1.Cuando la información inicial que se posee es insuficiente y de baja calidad, asi como cuando no se conocen suficientemente las tendencias de desarrollo correspondientes.

2.Cuando no pueden ser indentificados y rectificados a tiempo los crrores de concepción, de cálculo, etc., debito a limitaciones de tiempo, calificatión insuficente de los proyectistas u otras causas.

3.Cuando no se aplican los métodos, técnicas y procedimientos adecuados en el proceso de proyección y su evaluación.

Investigaciones realizadas por Teisseyre 14 refcridas a las limitaciones en la obtención de un efecto util total de las instalaciones industriales proyectadas y por Hernández Pérez Y Floss 6 vinculadas al cumplimiento de las exigencias de seguridad, proteccion e higiene del trabajo y del medio ambiente en diferentes proyectos de plantas industriales demuestran con hechos, como se provoca un aumento de los recursos materiales y financieros destinados a las inversiones respecto a lo planificado para obtener resultados técnicoeconómicos inferiores a los previstos, al mismo tiempo que ponen en evidencia que las tareas de proyecto pueden solucionares de una forna mas racional y eficiente si se aplican conscuentemente enfoques, procedimientos y técnicas modernas a esta compleja actividad.

Para poder evitar, eliminar y/o reducir los errores en un proyecto es necesario, en primer lugar, conocer los tipos de éstos para poder incidir de manera efectiva sobre las causas concretas que los originan Segun Rockstroh 10) los errores de un proyecto pueden clasificarse como se muestra en la Figura 1.

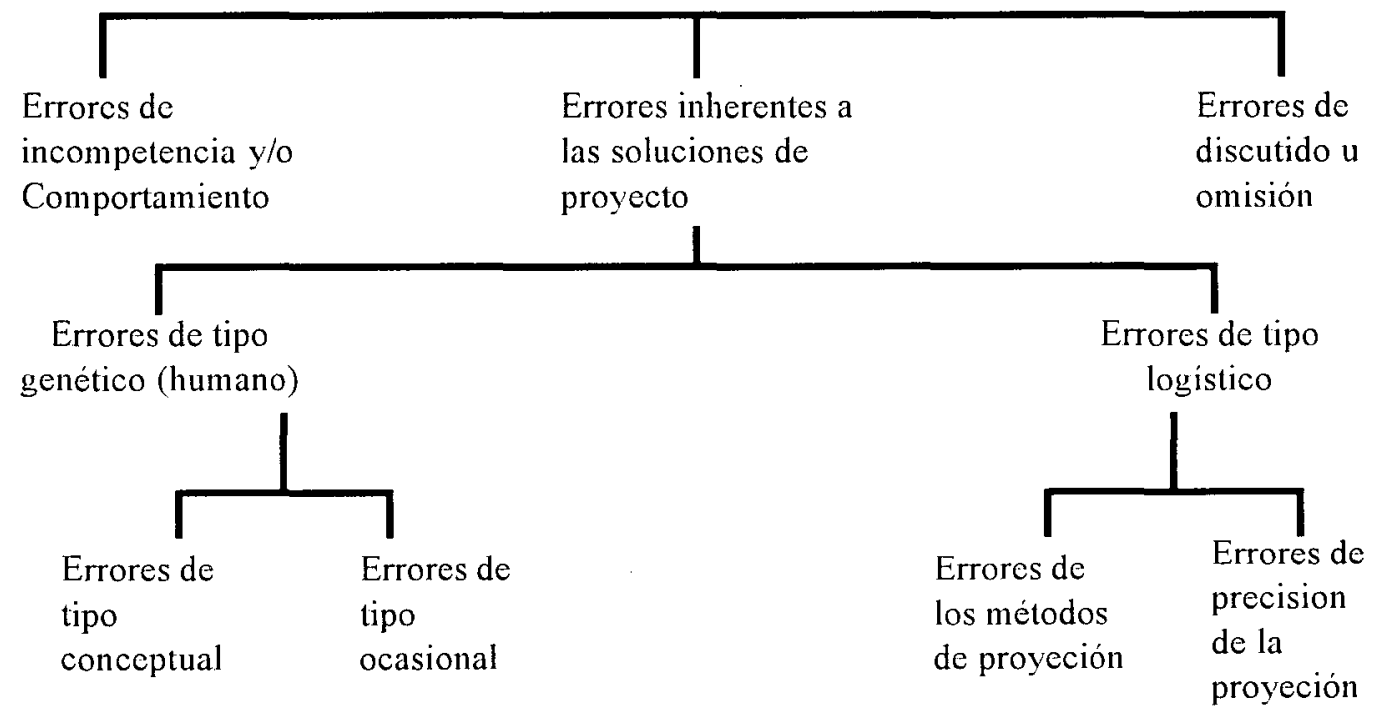

Figura 1. Classsificacion de los errores de un proyecto 10. 
Los errores de incompetencia o de omisión voluntaria son el resultado de una acción consciente directa o indirectamente que pueden tracr como consecuencia una disminución del rendimiento de las instalaciones, de la productividad del trabajo. etc. Ejemplos de este tipo de error lo constituyen la omisión de posibles variantes de solución, la sobrevalorición de posibles resultados a alcanzar en la instalación provectada con cl objetivo intensionado o no de "convencer" a personas, gremios, instituciones o entidades inversionistas o linancieras sobre las ventajas del proyecto.

Los errores por descuido o inadvertência son de indole formal y de carácter involuntario y se presentan, como regla gencral, debido a la utilización de métodos, expresiones de cálculo, equivocaciones de simbologia, utilización de medios auxiliares no apropiados a un caso concreto y otros.

Este fenómeno de aparición frecuente y que provoca. por ejemplo. que errores comctidos en otros proyectos se repitan innecessariamente o simplesmente, que no sean reconocidos como tales. subrava una vez más la ncccsidad del trabajo conjunto (interdisciplinario) en la concepción, elaboración y cvaluación de las soluciones de provecto asi como su perfeccionamiento a través de una decuada logística de provección.

Los errores iherentes a una solución de proyecto pueden ser de lipo humano (genético) y de ascguramiento o logistica de la proyección.

Los primcros se asocian fundamentalmentc a limitaciones o deficiencias en el grado de conocimicnto del problema a solucionar, asi como por insuficiente investigación y/o comprobación expcrimental fundamentalmentc en el caso de las nuevas inversiones. Estos, a su vez. pueden clasificarse en crrores de tipo conceptual y ocasionales. Segun Arldt 2, los primciros sc presentan fundamental en la partc técnico-tenológica de los proyectos cuando éstes se claboran a partir de supuestos erróneos o insuficientemente demostrados (fundamentalmente en las denominadas fases iniciales de las inversiones), asi como en la proyección de instalaciones fabriles de elevada complejidad en periodos de tiempo limitados o muy exigentes. Los de tipo ocasional se presentan principalmente a causa de la dinámica que caracteriza a los indices técnico-tecnógicos, organizativos y económicos de una solución de proyecto. En este sentido, el desconocimiento de las tendencias de desarrollo de cstos indices constituye una fuente frecuente de este tipo de erros. Tambien pueden introducirse cstos errores durante la elaboración de los proyectos al considerar determinadas informaciones como determinísticas, obviando su dinámica y/o aleatoriedad rcal, dadas por el propio carácter dinámico y estocástico que le cs inherente al proceso de producción 10. Con la creciente automatización del proceso de proyeccción es posible, de manera progresiva, incorporar esta tendecnia moderna en la proyección de instalaciones fabriles y con ello posibilitar, entrc otros aspectos, la fundamentación de reservas economicamente fundamentadas de las dimensiones básicas de las instalaciones que son objeto de provección o simplesmente, de un determinado tamano o capacidad de las instalaciones y por ende obtener soluciones cualitativamente superiores.

Los errores de tipo logístico en la proveccion se presentam como regla general, por la violación de la lógica de las investigaciones y tareas asociadas al proceso de proyección y su aseguramiento. Debido a que la selección de un detcrminado mćtodo de provección se 
encuentra generalmente prefijado (ver Seidel 13, los errores de tipo logistico no deben presentarse con mucha frecuencia en el trabajo de proyectistas experimentados y de empresas o firmas especializadas en esta actividad. Aunque no siempre la utilización de técnicas modernas de computo contribuye a clevar la calidad de los resultados de un proyecto, su no utilización puede contribuir a la aparición de errores de tipo logístico (sobre todo cuando la experiência de los proyectistas es limitada) y con ello a reducir el efecto util integral de una solución de proyecto.

Por otra parte no siempre los errores que se presentam en los proyectos tienen que sumarse y necesarimente conducir a un error $e$ consecuencia negativa final mayor. Junto a la lay de propagación de los errores debe considerase tambien el hecho de que éstos, en su efecto final conjunto tienden a compersarse $(+y-)$ a causa del mecanismo de autoregulación que se activa en todo proceso de proyeccion correctamente ejecutado 10. Además, y apoyando lo planteado debe senalarse que el propoio carácter cíclico de este proceso-analogamente puede ser comparado com la espiral de desarrollo de la calidad planteada por Juran [7] - contribuye también a la eliminación de los errores en las proyección o al menos, a la atenuación de sus efectos cn las diferentes fases y etapas sucesivas en que éste se lleva a cabo y que, de hecho, representam "niveles progresivos de calidad", en la elaboración de las soluciones de proyecto (ver Figura 2)

Figure 2. Niveles de calidad en la elaboración de las soluciones de proyecto (en analogia con lo planteado por Juran 7.)

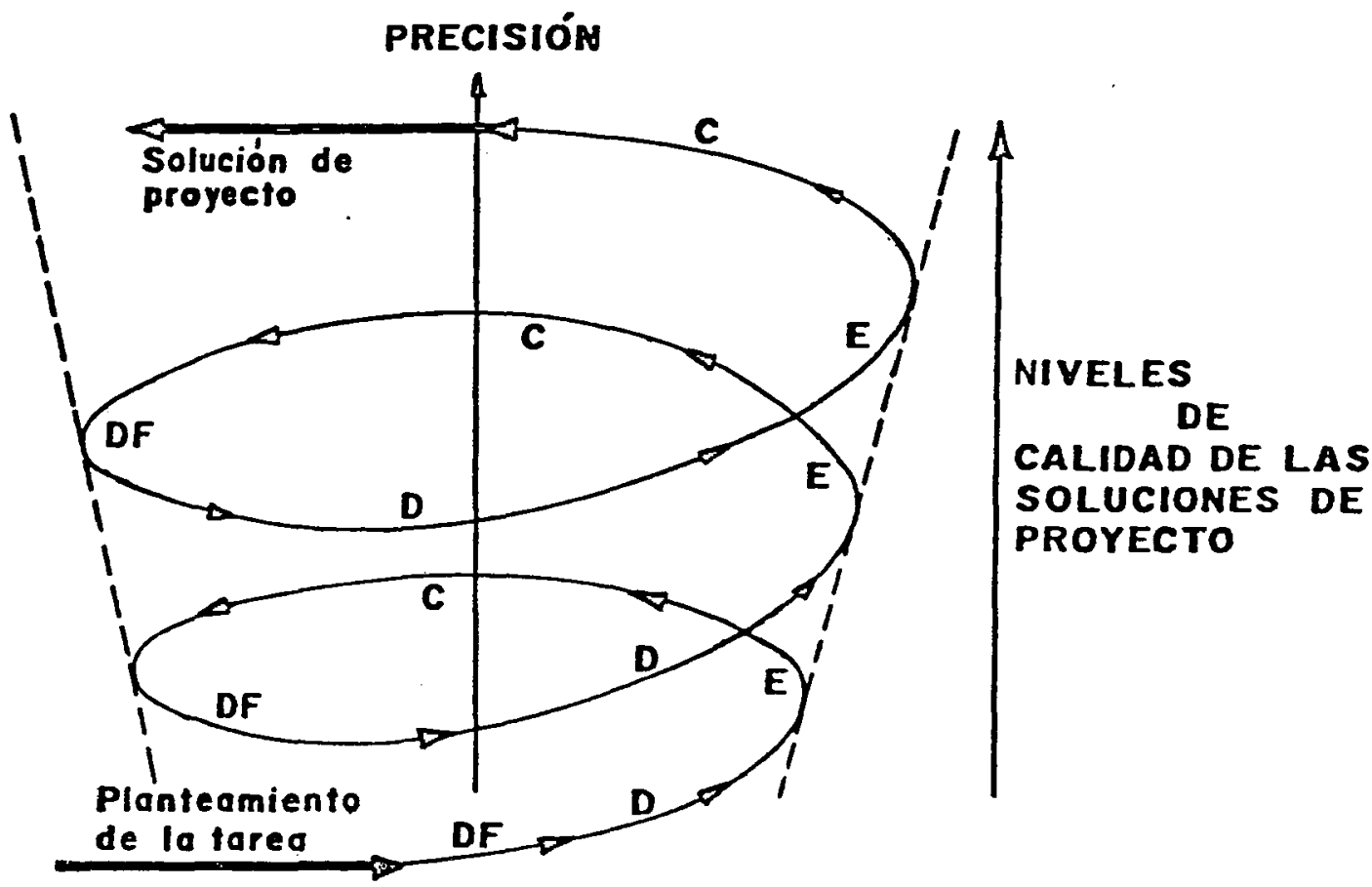

(DF: Determinación de funciones; D: Dimensionada; E: Estructuración; C: Conformación) 
De todo lo antes expuesto puede concluirse que mediante la incorporación de un enfoque sistmémico y un tratamiento cientifico riguroso del proceso de proyección que contemple, entre otros aspectos no menos importantes, una utilización más amplia y efectiva de las técnicas modernas de cómputo apoyadas en el desarrollo y aplicación de métodos estadisticos y de investigación operacional de carácter progresivo, los errores de todo tipo tienden a desaparecer o en el peor de lós casos, a disminuir sus efectos negativos y con ello obtener resultados cualitativamente superiores en las soluciones de proyecto.

\section{El Control del Proceso de Proyección, Evoluación de la Calidad de las Soluciones de Proyecto}

Para lograr una eliminación racional de los errores del proyecto, éstos tienen que ser reconocidos a tiempo y estimadas sus consecuencias.

Es por ello que al proceso de proyección de una instalación industrial en su totalidad debe asociarse un sistema exigente de control desde el inicio hasta su conclusión, incluyendo la evaluación final de la instalación industrial para su pucsta en marcha y explotación. La estructura básica de un sistema de este tipo se muestra esquemáticamente en la Figura 3 en analogia a lo planteado en [8] y [10].

La progresiva elavación de las exigencias de calidad de los proyectos en la actualidad, así como lo variado y complejo de su contenido como regla general, exige cada vez mas de una alta calificación del personal vinculado a este proceso, de una adecuada logística, así como de una dirección calificada, competente y debidamente organizada.

Si bien es cierto que las metodologais, leyes y regulaciones vigentes en nuestros pa-

Figura 3. Estructura básica de un sistema integral de control de la calidad de un proyecto (en analogia com $|8|, 10$ ).

Início del proyecto (planteamiento de la tarea)

\&

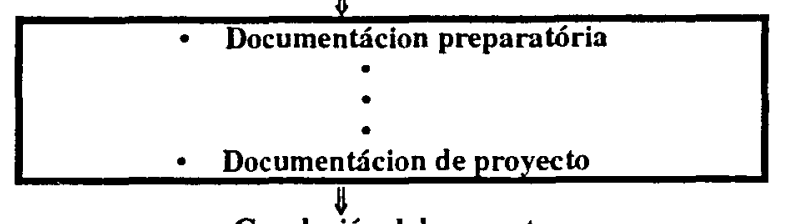

Conclusión del proyecto

$\Downarrow$

Ejecución del proyecto

$\Downarrow$

Pruebas del proyecto
$\Leftrightarrow \quad$ Control Inicial

\begin{tabular}{|c|c|}
\hline$\Leftrightarrow$ & $\begin{array}{l}\text { Controles } \\
\text { progressivos } \\
\text { a } \\
\text { soluciones } \\
\text { parcialesr }\end{array}$ \\
\hline & $\begin{array}{l}\text { Control final a la } \\
\text { documentación de } \\
\text { proyecto }\end{array}$ \\
\hline
\end{tabular}

$\infty \quad$ Controles progresivos y final a la ejecución del proyecto

$\Leftrightarrow \quad \begin{aligned} & \text { Evaluación final de la } \\ & \text { instalación para su } \\ & \text { puesta en marcha a la } \\ & \text { capacidad de éiseno }\end{aligned}$


ises vinculadas con la ejecucion del proceso inversionista cn su integralidad contemplan en su cstructura procedimientos de control que "safisfacen en térmicos generalés" las cxigencias de este proceso, existen aún insuficicncias en los métodos y procedimientos cmplcados para "medir" integralmente la calidad de las soluciones de proyecto en todas sus fases y clapas.

\section{Evaluación de la Calidad de las Soluciones de Proyecto}

La medida de la calidad del provecto de una planta industrial constituye de hecho, la basc para segurar los niveles de calidad exigidos en el discno de los productos elaborados en el proceso de prducción provectado, instalado y puesto en marcha.

Sobre la calidad de una solución de proyecto influyen múltiples y variados factores dado el carácter complejo de esta magnitud. En particular está determianda fundamentalmente por:

- la calidad de la documentación inversionista: o sea, cl cumplimiento de las especilicaciones de proyecto y su correspondencia con la cjecución de la obra;

- la calidad de las instalaciones proyectadas: o sea, máquinas, equipos e instalaciones, soluciones constructivas y arquitectónicas, viales, redes técnicas. servicios al personal, instalaciones de seguridad protccción e higiene de las personas, instalaciones fabriles y medio ambiente. ctc.;
- la calidad de las soluciones técnic-organizativas proyectadas para asegurar la cxplotación eficiente de las instalaciones a partir de su puesta en marcha.

Mientras de la calidad de las instalaciones y de las soluciones técnico-organizativas proyectadas se pueden ver influídas además por un grupo de factores "externos" al proceso de proyccción en si (por cjemplo, restricciones del mercado suministrador, disponibilidades de fucria de trabajo calificada, disponibilidades materiales. financieras y de la documentación inversionista cjerce una marcada influencia la "forma" (métodos, proccdimientos y medios) de proyectar y evaluar la calidad de las soluciones obtenidas (logística de la proyección).

Sobre los mćtodos, procedimientos y mcdio para clevar la racionalidad y eliciencia del proceso de proyección mediante un apoyo computacional adccuado, particularmente para instalaciones fabriles tipicas de la industria mecánica, se desarrollan trabajos investigativos en la Universidad Central de Las Villas, República de Cuba, con resultados ya disponibles algunos y alentadores otros.

En lo concernientc a la logística utilizada en el proceso de toma de decisiones y la evaluación de las soluciones de proyecto en todas sus lase, cl tipo y númcro de factores influyentes es, como regla gencral, elcvado. Tampouco es posible ni racional en la mayoria de los casos, dada la magnitud de estos sistcmas, resumir todos y cada uno de los factores influyentes en un metodo gencral. Solo aquellos que para un detcrm inado provecto se consideren como decisivos o fundamentales deben ser planteados y evaluados en la comparación de alternativas 17. Sin enbargo. en la práctica de la proyección han predomi- 
nado mas la aplicación de métodos de carácter económico para la evaluación de magnitudes denominadas indistintamente como "objetivas", "tangibles", o "economicamente cuantificables", sobre otros métodos de evaluación de propiedades técnicas de las soluciones para magnitudes consideradas como "subjetivas", "intangibles" o "no cuantificables económicamente" (o al menos de difícil cuantificación real), que han atentado contra la efectividad y racionalidad de las decisiones.

Si bien es cierto que el desarrollo alcanzado en los últimos anos en la aplicación de las técnicas de investigación operacional a la toma de decisiones, permite disponer en la actualidad de un instrumentário poderoso de procedimientos que pueden ser aplicados tambien con otros propósitos (por ejemplo, procedimientos multicriteriales 9 , se hace necesario tambien "rescatar" la aplicación de otros métodos considerados como convecnionales en la práctica de la proyección de plantas industriales; por ejemplo, métodos de evaluación por puntos, listas de chequeo, métodos de expertos, etc. En particular lo métodos de evaluación por puntos (o de ponderación) presentan condiciones propias para cuantificar el nível de calidad de una solución (Q), aunque debe prestarse la debida atención a la componente subjetiva de la evaluación (fijación de los factores de volaración y ponderación). Numcricamente puede calcularse el nivel de calidad de una variante de solución (Q) por la cxpresión general siquiente 10:

$$
Q_{k}=\left(\sum_{i=1}^{p} \mathrm{fw}_{i} / \sum_{i=1}^{\mathrm{m}} \mathrm{f} \mathrm{w}_{i(\max )}\right) * 100(\%)
$$

donde:

$f w_{i}=$ factor de valoración real de la cualidad " $i$ ", en puntos;

$$
\begin{aligned}
f \mathrm{w}_{\mathrm{i}(\text { mixi })}= & \text { factor de valoración máximo de la } \\
& \text { cualidad " } \mathrm{i} \text { (por ejemplo, } 5 \text { puntos } \\
& \text { en un sistema de }(0,5 \text { puntos) }
\end{aligned}
$$

Una variante de cálculo más completa de este indice, asociado a un método integral de evaluación de soluciones de proyecto es el planteado por Woithe y Muller en 17 que bajo la denominación de índice de calidad agregado (Qa) - determina para cada variante de solución " $k$ " por la expresión(4)

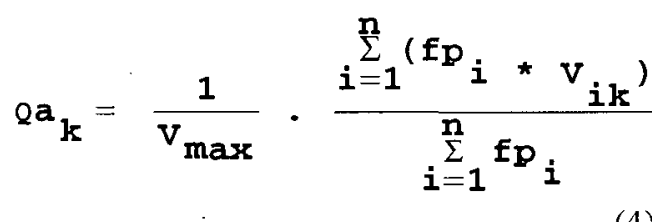

donde:

$\mathrm{V}_{\text {máx }}=$ valoración máxima que se otorga a cada calidad "i" cn el sistema empleado, en puntos (por cjemplo, 3 puntos en un sistema de 0. 3 puntos);

$\mathrm{V}_{\mathrm{ik}}=$ valoración otorgada a la calidad "i" cn la variante de solución " $\mathrm{k}$ ", en puntos (0 Vik 3);

$\mathrm{p}_{\mathrm{i}}=$ factor de ponderación figado para la cualidad "i" en el conjunto de cualidades a evaluar en las variantes de solución. En la literatura especializada se oferecen varias recomendaciones sobre diferentes sistemas deponderación; sin embargo en la práctica de la proyección se han obtenido buenos resultados aplicando un sistema donde: I fpi 5).

Variantes de estos métodos que incluyen procedimientos de ponderación, asi como 
aplicaciones comprobadas de su efectividad en diferentes actividades del proceso de proyección pueden consultarse en la literatura especializada; por ejemplo [11], [12] y [17].

\section{Fiabilidad en la Proyección de Plantas Industriales}

Cada instalación industrial proyectada y convertida en realidad tiene que cumplir con las funciones previstas para ćsta: por tanto, tiene que ser portadora de una determinada fiabilidad. La fiabilidad de una instalación industrial - tambien en analogia con un producto complejo - depende funcionamente de múltiples factores individuales y por tanto, posee propiedades cambiantes (dinámicas) que surgen del necesario incremento de las exigencias cualitativas como consecuencia del progreso cientifico-técnico del que éstas son portadoras en el periodo que media desde la fase de elaboración de los proyectos hasta la puesta en funcionamento de las instalaciones a plena capacidad y durante su vida útil [16].

La fiabilidad de un proceso de producción es tambićn una magnitud compleja, como complcjo es el carácter de éste y cuyos parámetros se pueden caracterizar segun Rockstroh 10 por:

- el tiempo de duración de su fucnión (po ejemplo, vide útil técnica y económica) $(\mathrm{Vu})$

- el ticmpo de duración de su función (por cjemplo, vida útil técnica y económica) $(\mathrm{Vu})$;
- el tipo y forma de utilización de los elementos individuales del proceso y su fiabilidad individual $(\mathrm{Ri})$;

- la "edad" y el estado técnico de las instalciones $(\mathrm{T})$;

- las necesidades de mantenimiento, servicios periodicos y reparación de las isntalaciones $(\mathrm{M})$.

O sea:

$R(s)=f(V, E, R i, T, M)$

donde:

$R(s)=$ fiabilidad del sistema en su conjunto.

Mediante una proyección "cualitativamente correcta" de las intalaciones industriales el proyectista puede influir decisivamente sobre su fiabilidad futura desde las mismas fases de preparación de las inversiones. El concepto de proyección cualitativamente correcta vincula estrechamente los aspectos sobre calidad de la proyección antes expuestos con los relativos a la fiabilidad de las instalaciones 5 y [11].

Para alcanzar un nivel adecuado de fiabilidad de las instalaciones industriales proyectadas se requicre considerar medidas que garanticen los trabajos de mantenimiento y renovación del parque de equipos, entre otras no menos importantes. Con cl crecimiento sostenido de la mecanización y automatización de los procesos productivos se eleva la complejidad de las operaciones tecnológicas y no tecnológicas y con ello se plantean cada dia mayores exigencias a la capacidad de las instalaciones de mantenimiento en las plantas industriales que son objeto de proyección [4]. 
Este hecho debe, incluso, ser en el futuro mas considerado en la actividad de proyección que en la actualidad para evitar omisiones que puedan conducir a gastos adicionales en la operación de las instalaciones.

Cada medida que contribuya a elevar la fiabilidad individual de cada elemento, instalación o proceso parcial contribuye a incrementar la fiabilidad del sector productivo, taller y/o de la instalación fabril en su conjunto y com ello, satisfacer de manera mas cabal las necesidades crecientes de bienes materiales de la sociedad. Un grupo de estas medidas a considerar en la fase de proyección de las instalaciones industriales en analogia a las planteadas por Rockstroh [10] y por Woithe y Muller [17] son:

claborar cronogramas ágiles y realistas para las diferentes fases y etpas del proceso inversionista sobre la base de una máxima reducción del tiempo que media entre la concepción de las soluciones y su puesta en marcha y mantener un control riguroso y sistemático de su ejecución;

seleccionar en lo posible instalaciones, máquinas, ctc, de elevado rendimiento productivo y con una alta fiabilidad individual que, a su vez, puedan adaptarse con racionalidad a posibles variaciones de los programas de producción;

garantizar las capacidades e instalaciones necesarias para el mantenimiento y reparación de las instalaciones productivas, asi como la modernización y renovación a tiempo de las mismas sobre la base de una adecuada estrategia;

selecionar $y / o$ disenar e instalar $y / o$ construir instalaciones fabriles de uso general (por ejemplo, edificaciones, redes técnicas, sistemas y medios de transporte y manipulación, etc.), que garanticen una adecuada flexibilidad ante los cambios en la producción, que no presenten una alta variedad en tipos diferentes y que demanden relativamente pocos gastos de mantenimiento;

asegurar el rendimiento total exigido en determinados procesos tecnológicos u operaciones críticas o fundamentales mediante la creación de reservas economicamente fundamentadas que contribuyan a garantizar racionalmente la continuidad de trabajo necesaria de las instalaciones;

considerar en el proyecto instalaciones de protección contra incendios (detección y extinción), protecciones eléctricas (incluyecndo la protección contra descargas eléctricas) y de proteción del medio ambiente;

eliminar toda posibilidad de errores de operación a través de una adecuada selección y capacitación del personal que operará la planta, la elaboración de tecnologias de control, indicaciones para el personal, etc.

\section{Conclusiones y Recomendaciones}

El proyecto de una planta industrial, asi como esta última, considerada como una instalación real, constituyen un "proceso" y un "producto complejo" respectivamente, a los que se asocian, requerimientos, caracteristicas, especificaciones e indices de calidad y fiabilidad y como tales deben ser enfocados para "asegurar su calidad" como base fundamental para el aseguramiento de la calidad de las futuras producciones que saldrán de sus instalaciones productivas. 
Sc ratifica la necesidad de concebir y/o perfeccionar un procedimiento integral de evaluación de la calidad de los proyectos de plantas industriales desde las denominadas fases iniciales de las inversiones hasta su puesta en funcionamicnto a la capacidad de diseno, con un enfoque y logística que se corresponda cada vez más con las tendencias mas actuales en este campo y luchar por su "compatibilidad" a nivel de nuestras economias como base de la necesaria y no muy lejane integración latinoamericana. En este sentido debe prestarse especial atención a la calificación del personal encargado de este proceso y su adecuada direción, así como a las vias mas racionales para el reconocimiento $y$ eliminación de los errores que pueden presentarse en el proceso de proyección.

En este perfeccionamiento debe prestarse tambien especial atención a la precisión de las características, especificaciones e indices de calidad y liabilidad que deben caracterizar una solución de proyecto de una planta industrial, incluyendo con particular énfasis, aquellas que cjerzan influcncias decisivas sobre el medio ambiente.

Para garantizar instalaciones industriales de alta confiabilidad productiva se precisa la adopción de medidas concretas que deben ser consideradas y precisadas progressivamente en todas las fases del proceso de proyección. En el marco de estas medidas debe enfatizarse en aquellas que contribuyan al ascguramiento de las capacidades de mantenimiento y reparación de las instalaciones industriales.

\section{Notas al Texto (página 1)}

(1). Término utilizado para denominar de manera general a las medidas de racionalización, nodernización, ampliación y reconstrucción propiamente dicha de las instalaciones industriales.

\section{Referências}

ALMEIDA, H. S. \& TOLEDO, J. C. (1991) Calidad Total del Producto. Revista Producción (ABEPRO), Vol. 2, No. 1, Rio de Janeiro, pp. 20, 37 (en portugués).

ARLDT, H. (1970) - Sicherung der Schutzgute bei der Vorbereitung und Durchfuhrung von Investitionen. Verlag Tribune, Berlin.

CROSBY, P. B. (1987) - What are requeriments? The successful organization takes them seriously. Quality Progress, Vol. XX, N8, p. 47., USA.

GUARDIA DAQUIN, J. A. \& HERNÁNDEZ PÉREZ. G. (1988). Efectividad del mantenimiento en empresas industriales - Sistema de actividades para su proyección. Revista Ingenieria Industrial (ISPJAE), Vol. IX, N"2, ciudad de La Habana, pp. 109-116.

HENNING, D. \& Franke, L. (1987). Zuverlãssigkeitsorientierte Projetierung von Fertigungsstrukruren im Maschinenbau. Fertigungstechnik und Betrieb. J.g. 37 , Nr. 6, Berlin, S. 359,362.

HERNANDEZ PÉREZ, G. \& FLOSS, D. (1985) - Exigencias de la organización cientifica del trabajo en el proyecto de industrias. Revista lngenieria Industrial (ISPJAE), Vol. VI, Nº. 3. Ciudad de La Habana, pp. 252-260. 
JURAN, J. M. et alii (1974) - Quality Control Handbook (Sect. 2), Third Edition. Mc Graw Hill Co. New York, pp. 2.4, 2.10.

KUME, H. (1980) - Formal Design Review. UNIDO-AOTS, UN-QC, Tokyo, pp. 8, 30 .

MONTEIRO GOMES, L. F. A. \& DUARTE, A. M. Jr. (1991) - La cvaluación de proyectos con múltiples criterios. Revista Producción (ABEPRO). Vol. 2. N*. 1, Rio de Janeiro, pp. 5.19 (en portugués).

OITHE, G. \& HERNÁNDEZ PÉREZ, G. (1986) - Fundamentos de la Proyección de Fábricas de Construcción de Maquinaria. (1 Edición). Editorial Pueblo Y Educación, Ciudad de La Habana, pp. 16,18 .

ROCKSTROH, W. (1980) - Die technologische Betriebsprojektierung, Band 1: Grundlagen und Methoden der Projektierung. VEB Verlag Technik, Berlin.

ROCKSTROH, W. (1980). Die Technologische Betriebsprojektierung Band 4: Projektierung des Industriebetriebes. VEB Vcrlag Technik, Bcrlin.
REED, R. Jr. (1969). Plant Layout-Factors, principles and techniques. Ediciones "R". Instituto Cubano del Libro. Ciudad de La Habana. pp. 352, 367.

SEIDEL, H. (1980) - Anforderungen der Industrie an Effektive Projektlosungen. Fertigungstechnik und Betrieb, Jg. 30, No. 2, Berlin, S. 100-102.

TEISSEYRE, W. L. (1966) - Klassifisicrung von Projektierungsfehlern und deren Einfluss auf zu erreichende okonomische Ergebnisse. Prezglad Mechaniczny, Heft. 3 (traducción alemana del original en Polaco).

WOITHE, G. \& GOTTSCHALK, E. (1976). Flexibilitat und Variabilitat von Maschinenbaubetrieben. Fertigungstechnik und Betrieb. Jg. J6, Heft. 12, S. 706, 710 .

WOITHE, G. \& MULLER, G. (1982) - Beurteilung von Projektlosungen autonomer Strukturen. Miss. Zcitschrift der T. H. "Otto von Guerickc", Magdeburg, Jg. 26. Helft 5. S. 91, 93.

WOITHE, G. ct alii (1979) - Zur Flexibilitat von Maschinenbautetricben. Dic Technik, $32 \mathrm{Jg}_{\mathrm{g}}$. Heft 7. S. 373,374 